\title{
KULEUVEN
}

\begin{tabular}{|c|c|}
\hline Citation & $\begin{array}{l}\text { Diana Ugryumova, Gerd Vandersteen, Bart Huyck, Filip Logist, Jan van Impe, } \\
\text { and Bart De Moor, (2013) } \\
\text { Identification of a Non-Insulated Distillation Column From Transient } \\
\text { Response Data, } \\
\text { IEEE Transactions on Instrumentation and Measurement, 62(5), 1382-1391. }\end{array}$ \\
\hline Archived version & $\begin{array}{l}\text { Author manuscript: the content is identical to the content of the published } \\
\text { paper, but without the final typesetting by the publisher }\end{array}$ \\
\hline Published version & http://dx.doi.org/10.1109/TIM.2012.2234577 \\
\hline Journal homepage & http://ieeexplore.ieee.org/xpl/Recentlssue.jsp?punumber=19 \\
\hline Author contact & $\begin{array}{l}\text { filip.logist@cit.kuleuven.be } \\
+32(0) 16322703\end{array}$ \\
\hline IR & https://lirias.kuleuven.be/handle/123456789/398776 \\
\hline
\end{tabular}

(article begins on next page) 


\section{Identification of a Non-Insulated Distillation Column From Transient Response Data}

\author{
Diana Ugryumova*, Gerd Vandersteen \\ Vrije Universiteit Brussel \\ dept. ELEC, Pleinlaan 2, 1050 Brussels, Belgium
}

\author{
Bart Huyck ${ }^{\ddagger}$, Filip Logist ${ }^{\ddagger}$, Jan Van Impe , Bart De Moor $^{\S}$ \\ Katholieke Universiteit Leuven \\ dept. CIT, W. de Croylaan 46, 3001 Heverlee, Belgium ${ }^{\ddagger}$ \\ dept. ESAT, Kasteelpark Arenberg 10, 3001 Heverlee, Belgium ${ }^{\S}$
}

\begin{abstract}
Distillation is the most used separation technique worldwide. However, it is also an energy intensive method. A way to reduce the energy consumption is to optimize the working conditions of distillation processes. This paper uses system identification to find an accurate model of a distillation column that can be used in, for example, model predictive control. Identification of a pilot-scale distillation column is done in the frequency domain in two steps. First, a non-parametric local polynomial method is used to eliminate the leakage effects and to estimate the frequency response and noise contributions. A fast and a robust local polynomial methods are compared. Second, results from the first step are used to find a low-order rational Laplace model with a time-delay term. Due to lack of insulation, the system's operating point is varying with ambient temperature. By using a reparametrization of the initial model, it is possible to account for these changes. The final result is a metamodel which depends on the frequency and the effective separation heat of the column. In the future, this model can be applied in model predictive control to optimize the working conditions of a distillation column. Simulation data and real measurements on a pilot-scale distillation column are used to illustrate the results.
\end{abstract}

Index Terms-Identification, frequency response, time-varying systems, transient response.

\section{INTRODUCTION}

Distillation is the most used separation technique worldwide and is very energy consuming. Hence, it is important to reduce energy consumption as much as possible.

An efficient way of reducing energy consumption in distillation columns is by using accurate models. This helps to predict and control the distillation processes, and therefore helps to reduce the energy consumption.

Several so-called white-box models of distillation columns exist, which make use of balance equations for material and energy, flow dynamics, etc. A good overview of the status of dynamic modeling and control of the distillation columns before 1997 is given in [1]. This survey paper clearly pointed out some important identification problems, e.g., (i) the need for a multiple-input-multiple-output (MIMO) model directly from the measurements, which accounts for directionality in the process and (ii) a model which accounts for the disturbances in a simple manner. Also, it seems from the literature that identification of distillation columns mostly uses steadystate response data. Using these methods on the transient

\footnotetext{
* Corresponding author. Email: diana.ugryumova@vub.ac.be, telephone
} number: +32 (0)26292949 response data gives a biased estimate of the systems dynamics. In addition, the white-box models are in practice often cost and labor intensive to calibrate.

New techniques were used over the last fifteen years to model distillation columns. For example, a nonparametric modeling technique like artificial neural networks takes explicitly into account the physical couplings in the column [2]. The drawback is that the neural networks are approximate models. Also, a good understanding of these models is needed for a practical usage of the models. To account for the nonlinearities in the distillation processes, a Wiener model can be used [3]. This identification is suitable for a closed-loop set-up.

The goal in this paper is to find an accurate but simple blackbox model of a distillation column to be used, for example, in model predictive control. A review on the use of the model predictive control in industry can be found in [4].

There are several challenges in the identification of a distillation column. These include: (a) large time constants, (b) variations due to ambient temperature changes, (c) constraints on the operation and excitation of the column, (d) measurement noise, (e) nonlinearities, (f) time-delays in the response of the column, and (g) the ill-conditioning of the system [5], [6].

(a) A long transient response (large time constant) in the time domain translates into leakage in the frequency domain. Because of leakage errors, the frequency response function (FRF) estimate is biased when using frequency-domain system identification methods [7].

(b) The varying ambient temperature causes the system's operating point to change. Thus, we cannot use the same model for different ambient temperatures.

(c) Because of safety reasons, there are certain constraints on the operation of the column. Also, several limitations of the column's excitation exist. For example, the duty of the reboiler has to be above a certain minimum for the separation of liquids to take place. At the same time, the reboiler power may not exceed a certain level if the less volatile component has to remain at the bottom of the column.

(d) Measurement noise is always present due to imperfect measurement instruments. Here, we assume that there is noise present on both the input and the output measurements (errorsin-variables framework [7]).

(e) A high-purity distillation column is believed to be strongly nonlinear system. This should be taken into a con- 
sideration when selecting a model structure for the distillation column. The pilot-scale distillation column we use is not a high-purity column, allowing us to use a linear model to estimate the column.

(f) Time-delays are present in a distillation column, for example, due to its recycle loops. The time-delays of the pilotscale column used are in the order of several minutes.

(g) The ill-conditioning of the system means that it has strong directionality [5], [6], i.e. the condition number of the gain matrix is (too) large. In other words, this means that the processes inside a distillation column are strongly interactive. Although this is believed to be a major problem in distillation column identification, we will not address the problem here.

In this paper, we address several of the above mentioned identification problems for distillation columns ((a), (b) and (d) to (f)). Namely, we eliminate the leakage error and estimate the level of nonlinearities and noise using nonparametric identification methods. In addition, we also find a parametric low-order rational-form model in the Laplace domain with a time-delay that depends on the ambient temperature. To solve the leakage problem and to estimate the nonparametric FRF together with its error bounds, we use a local polynomial method (LPM). The choice of the system's excitation also plays an important role. Here, we use an optimized randomphase multisine excitation and make sure that it meets the constraints of the column. The LPM eliminates the transient by first fitting a polynomial in the frequency domain to the nonexcited frequencies followed by a transient removal step. Then, using the nonparametric estimate of the FRF, we find a low-order rational-form model with time-delay for the system's FRF. Finally, a simple expedient on the reboiler power is used that makes the estimation of a model with parameters depending on the ambient temperature easier. Thus, the goal is to estimate a metamodel of distillation column whose parameters depend on the ambient temperature.

To summarize, the contributions of this paper are the following:

- An identification of distillation columns from transient response data is possible which leads to shorter measurement times.

- The approach results in a MIMO transfer function estimate.

- Estimation of the level of nonlinear distortions is possible.

- A metamodel is presented to account for the changes in the set-point of the distillation column due to the changes in the ambient temperature.

It should be noted that all these results can be accomplished without much interaction with the user.

The paper is structured as follows: first, the identification methods to model a distillation column are explained in Section II. Subsection II-A defines a multisine excitation, while the LPM is explained in Subsection II-B. Parametric modeling is mentioned in Subsection II-C. How to handle temperaturedependence of our system is explained in Subsection II-D. Then, in Section III, a simulation of a white-box model of a pilot-scale binary distillation column, originally developed by [8] and [9], is used to demonstrate the method. Finally, we present the results using real measurement data from a pilot-scale distillation column at KUL-BioTeC in Section IV.

This paper extends the results presented in [10] with the following items:

- A theoretical background on the recently developed fast local polynomial method [11] is added. In this way, the usage of the local polynomial method can be compared for the fast and the robust LPM's.

- A simulation analysis is made for a single-input-multipleoutput (SIMO) and a MIMO systems of a white-box model of a distillation column. In both cases the performances of the fast and the robust methods are compared.

\section{IDENTIFICATION AND MODELING APPROACH}

A distillation column is considered to be a MIMO nonlinear system whose system dynamics can be affected by the changes in ambient temperature. In this research, we consider an uninsulated pilot-scale distillation column which is not in steady-state; we therefore have to deal with leakage in the frequency domain.

In this section, we explain the identification and modeling methods we use to eliminate the transient, model the varying dynamics due to the ambient temperature, estimate the nonparametric frequency response matrix (FRM) and give an indication of the level of nonlinearities.

Optimized random-phase multisine inputs are used together with the LPM to obtain better nonparametric estimates of the FRM and its variance. This nonparametric FRM is used to fit a low-order rational-form parametric model. To account for the changes in ambient temperature, we propose that the parameters of the model vary as a function of the difference between the ambient temperature and the total heat loss of the column.

\section{A. Multisine excitation}

Orthogonal random-phase multisine inputs are used to optimally excite the column [12]. Multisines are periodic signals, which in our case have the same amplitude $a_{i}$, the same sample period $T_{s}$ of 1 second, the same number of excited frequencies $F$, fundamental frequency $f_{0}$ and zero mean random phase $\phi_{k, i, r}$ for each excited frequency $k$, input $u_{i}$ and realization $r$ :

$$
u_{i, r}(t)=\sum_{k=1}^{F} a_{i} \cos \left(2 \pi f_{0} k t T_{s}+\phi_{k, i, r}\right)
$$

Orthogonal random-phase multisines are several realizations of multisines where the matrices of phases for each realization are unitary matrices. The advantage of these orthogonal multisines is their guaranteed optimal conditioning of the input matrix that is used to compute the nonparametric FRM [12]. 


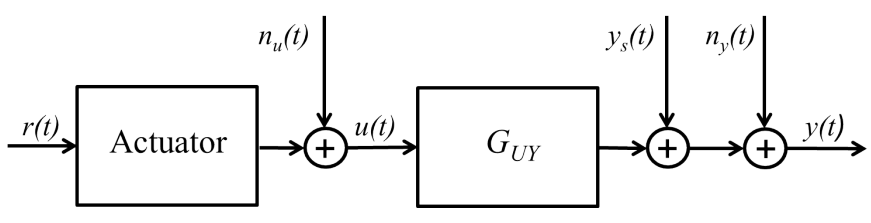

Fig. 1. A schematic representation of the considered system. The following signals are indicated in time-domain: $r$ the reference, $n_{u}$ additive input noise $u$ the input, $y_{s}$ the nonlinear distortion, $n_{y}$ additive output noise, $y$ the output. The frequency response of the system is indicated by $G_{U Y}$.

\section{B. Local polynomial method}

A short description of a recently developed nonparametric identification technique called LPM [11] is given in this subsection. There exist two variations: the so-called 'fast' and 'robust' LPM's. Both methods use a periodic excitation and:

1) remove the transient,

2) estimate the nonparametric FRM,

3) determine the variances of FRM that are due to noise and stochastic nonlinearities.

Both methods use a two-step procedure. The first step estimating and eliminating the transient term - is done in a similar way in both fast and robust LPM's. This step is explained in this subsection. The second step of estimating the FRM is different for the two methods and is explained in Subsections II-B1 and II-B2.

Here, we consider a general errors-in-variables case with noise on the input and the output, see a schematic representation in Fig. 1. To be able to estimate the transient and noise on the input the LPM assumes that the reference signal is known. In case that the noise on the input is negligible, the following method can be used without knowing the reference signal and substituting the input for the reference.

Both fast and robust LPM's start with the knowledge that the spectra satisfy:

$$
\begin{aligned}
& U(k)=G_{R U}\left(\Omega_{k}\right) R(k)+N_{U}(k)+T_{G_{U}}\left(\Omega_{k}\right), \\
& Y(k)=G_{R Y}\left(\Omega_{k}\right) R(k)+Y_{s}(k)+N_{Y}(k)+T_{G_{Y}}\left(\Omega_{k}\right),
\end{aligned}
$$

where $X=R, U, Y$ is a Fourier transform of $n_{u}$ reference and $n_{u}$ input, and $n_{y}$ output sequences, respectively; $\Omega_{k}$ is the generalized frequency at the $k$-th frequency line; $G_{R U}(\Omega)$ and $G_{R Y}\left(\Omega_{k}\right)$ are the $n_{y} \times n_{u}$ FRM's from the reference signal to the input and from the reference signal to the output, respectively; $N_{U}$ and $N_{Y}$ are the noise terms; $Y_{s}$ is the zero mean stochastic nonlinear distortion; $T_{G_{U}}$ and $T_{G_{Y}}$ are the transient terms of the system. The FRM of the system $G_{U Y}$ is computed in the following way:

$$
G_{U Y}=G_{R Y}\left(\Omega_{k}\right) G_{R U}^{-1}\left(\Omega_{k}\right) .
$$

In this case, $G_{U Y}(\Omega)$ is the best linear approximation of the system, in mean square sense [13].

We use several periods $P$ of a random-phase multisine signal to excite the system. At least two periods of a periodic excitation are enough. Transforming this to the frequency domain, we know exactly which frequency lines are excited $Y(k P)$ and which are nonexcited $Y(k P+r)$ with $r$ not a

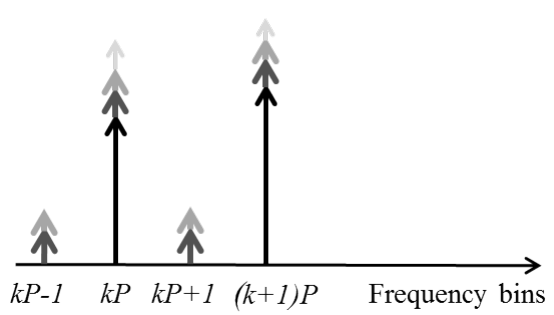

Fig. 2. A frequency domain representation of a periodic measurement with transient present. (black) frequency response on the excited lines, (gray to light gray) transient, noise and nonlinearities, respectively.

multiple of $P$. The excited frequency lines $k P$ contain the information about frequency response $G$, the nonlinearities $Y_{s}$, the transient $T_{G_{Y}}$ and the noise $N_{Y}$. Only the transient and the noise contributions are present on the nonexcited lines $k P+r$, such that:

$$
\begin{aligned}
& Y(k P)=G_{R Y}\left(\Omega_{k P}\right) R(k P)+Y_{s}(k P)+N_{Y}(k P)+T_{G_{Y}}\left(\Omega_{k P}\right), \\
& Y(k P+r)=N_{Y}(k P+r)+T_{G_{Y}}\left(\Omega_{k P+r}\right) .
\end{aligned}
$$

A visual representation of this is given in Fig. 2. We assume that the system is a period in, same period out [13]. That is why $Y_{s}$ appears only on the lines $k P$ and not on the lines $k P+r$ with $r$ not a multiple of $P$. Thus, noise and nonlinear distortions can be separated when multiple periods are measured, as shown in the rest of this section. However, if you are not interested in detecting the nonlinear distortions (e.g. in case of linear or weakly nonlinear device), then the measurement time can be reduced with the drawback that the nonlinear distortions and the noise cannot be separated anymore.

In the first step of the LPM, the transient terms are locally approximated and then removed [11]. This comes from the knowledge that the transient in frequency domain is a smooth function of the frequency. Thus, we can approximate the transient $T(\Omega)$ by a polynomial of the frequency, as in:

$$
T\left(\Omega_{k P+r}\right)=T\left(\Omega_{k P}\right)+t_{1}(k) r+t_{2}(k) r^{2}+t_{3}(k) r^{3}+\ldots
$$

This polynomial should be at least of order two [13]. In this way, the nonexcited lines are used to locally fit a polynomial to the transient terms and estimate the noise covariance. Next, the estimated transient term is subtracted from all the excited lines, resulting into $\hat{Y}$, an estimate of the output signal $Y$ with transient removed:

$$
\hat{Y}(k P)=G_{R Y}\left(\Omega_{k P}\right) R(k P)+Y_{s}(k P)+N_{Y}(k P) .
$$

Exactly the same reasoning holds for the input signal $U$. Thus, using the above steps it is possible to estimate and eliminate the input transient term $T_{G_{U}}$ and estimate the input noise $N_{U}$. For further details see [11], [13].

Eq. (6) is used in Subsections II-B1 and II-B2 as a starting point to find a nonparametric FRM estimate. 
1) The fast local polynomial method: only uses one realization of a random-phase multisine excitation, as in Eq. (1) with $i=1$ and $r=1$. It only needs at least two periods of the multisine. This is where the name 'fast' comes from.

Assume that the FRM $G(\Omega)$ is also a smooth function of the frequency. Thus, the fast LPM assumes that both the transient and the frequency response function can be approximated locally by a low-order polynomial in frequency. Like for the transient term estimation, at least a second order polynomial is used to find the nonparametric FRM:

$$
\begin{aligned}
G\left(\Omega_{k P+r P}\right) & =G\left(\Omega_{k P}\right)+g_{1}(k) r P+g_{2}(k)(r P)^{2}+\ldots \\
& =\hat{\Theta}\left[\begin{array}{llll}
1 & r P & (r P)^{2} & \ldots
\end{array}\right]^{T}
\end{aligned}
$$

where both $k P$ and $r P$ indicate the excited frequency lines, $\hat{\Theta}$ denotes the vector of unknowns which we want to determine and $x^{T}$ denotes a vector transpose.

Combining Eq. (6) and (7) for several excited frequency lines $n_{\mathrm{LPM}}=k P-r P, \ldots, k P+r P$ and using a least squares approximation to find $\hat{\Theta}$, we find:

$$
\hat{\Theta}=\hat{Y}_{n} K_{n}^{H}\left(K_{n} K_{n}^{H}\right)^{-1}
$$

where a numerically stable method is used to compute the inverse of $K_{n}$, which has the following structure:

$$
\begin{aligned}
& K_{n}= \\
& \left(\begin{array}{rccll}
R(k P-r P) & \cdots & R(k P) & \cdots & R(k P+r P) \\
(-r P) R(k P-r P) & \cdots & 0 & \cdots & (r P) R(k P+r P) \\
\vdots & & \vdots & & \vdots \\
(-r P)^{o_{\mathrm{pol}}} R(k P-r P) & . . & 0 & \cdots & (r P)^{O_{\mathrm{pol}}} R(k P+r P)
\end{array}\right)
\end{aligned}
$$

The frequency lines $n_{\mathrm{LPM}}$ are chosen symmetrically around the excited frequency of interest $k P$, if possible.

For a polynomial of order $o_{\text {pol }}$, the size of matrix $K_{n}$ is $\left(o_{\text {pol }}+1\right) n_{u} \times n_{\text {LPM }}$. This matrix can become ill-conditioned for large orders of $o_{\text {pol }}$.

2) The robust local polynomial method: is used in combination with an orthogonal multisine excitation to preprocess the data. It uses $n_{u}$ experiments for each realization. At least two realizations are needed to differentiate between $Y_{s}$ and $N_{Y}$. As a result, different realizations are used to calculate the average FRM and the total covariance on the FRM estimate, $Y_{s}+N_{Y}$. For more details about LPM, see [11], [13]. This method is called 'robust' because no further assumption is needed about the FRM.

The similarities and the differences of fast and robust LPM's are summarized in the table below. Here, $N$ indicates the length of one period of the excitation signal used, $N_{R}$ is the number of realizations needed only for the robust LPM. The variance of the estimated nonparametric FRM is denoted by $\sigma^{2}$ and is due to the noise and the nonlinearities. The total measurement time is denoted by $t_{\text {meas }}$.

\begin{tabular}{l|l|l} 
& Fast & Robust \\
\hline LP approx. transient & + & + \\
LP approx. FRM & + & - \\
$\sigma^{2}$ due to $N_{Y}$ and $Y_{s}$ & + & + \\
$t_{\text {meas }}$ & $N P, P \geq 2$ & $N P \times N_{R} n_{u}$, \\
& & $P \geq 2, N_{R} \geq 2$
\end{tabular}

Both methods approximate transient by a local polynomial and both can differentiate between the variances due to noise and stochastic nonlinearities. The two LPM's differ in the calculation of FRM and the total measurement time $t_{\text {meas }}$. For equal $t_{\text {meas }}$, the fast LPM gives an FRM estimate with a higher resolution than the robust LPM.

\section{Parametric model}

Eventually, our goal is to obtain a parametric model of the distillation column. Thus, using the nonparametric FRM estimate and its (total) variance, found with LPM, we make a parametric model of our system using MATLAB function elis from the fdident package [14]. This model describes the distillation column by its poles and zeros and a time-delay term.

Initially, these parametric models are obtained for a constant ambient temperature. By varying the ambient temperature, the operating point of the system changes. Thus, we estimate a parametric model for several operating points of the system.

\section{Temperature varying system}

Distillation columns are often not or not sufficiently insulated from their environment. The amount of heat lost over the whole column during the operation is directly related to the ambient temperature. This relation is shown in the following equation:

$$
Q_{\text {loss }}\left(T_{\text {ambient }}\right)=\sum_{i=0}^{\# \text { Trays }} S_{i} \cdot C_{\mathrm{HT}} \cdot\left(T_{\text {tray }_{i}}-T_{\text {ambient }}\right),
$$

where $S_{i}$ is the outer surface of the $i^{\text {th }}$ part of the column, $C_{\mathrm{HT}}$ is a coefficient of the heat transfer between the distillation column and the ambient environment, $T_{\text {tray }_{i}}$ is the temperature on tray $i$, where $\operatorname{tray}_{i=0}$ is the reboiler, and $T_{\text {ambient }}$ is the environmental temperature. Hence, the ambient temperature has an impact on the operation of the distillation column.

This impact of the ambient temperature can be significant for small pilot-scale columns with a large surface-to-volume ratio, whereas the impact is less pronounced for large industrial columns with a small surface-to-volume ratio. To enable an enhanced control, models can incorporate changes due to the ambient temperature.

Initially, the idea for a model structure that accounts for the ambient temperature was a model with parameters and delay term varying with ambient temperature. We denote such a model as $\mathcal{M}\left(Q_{\text {loss }}\left(T_{\text {ambient }}\right), Q_{\text {reboiler }}\right)=\mathcal{M}\left(T_{\text {ambient }}\right)$, where $\mathcal{M}(\cdot)$ is a model structure depending on certain parameters, $Q_{\text {reboiler }}$ is a duty of the reboiler, which in this case is constant. As mentioned before, to find an accurate model of 

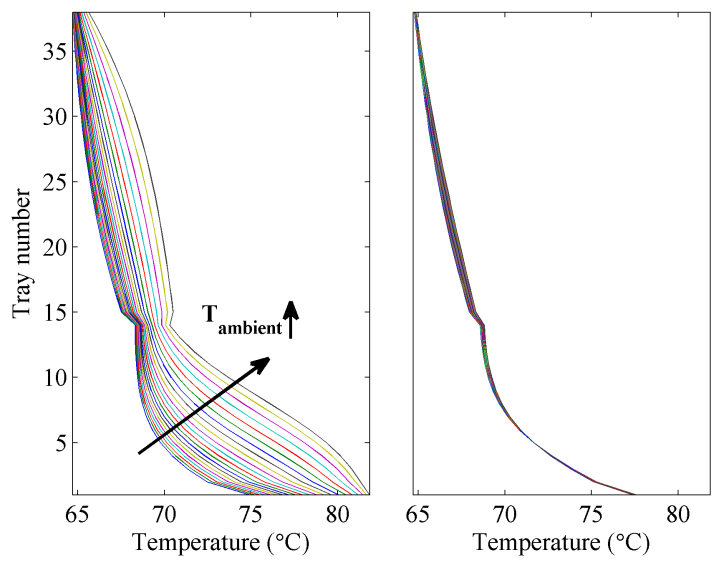

Fig. 3. Plot of the temperature inside the column as a function of the tray number; to the left: operation of the column varies with ambient temperature to the right: the reboiler power is adjusted to the variations in the ambient temperature such that the operational temperatures of the column become more or less constant.

the distillation column, it is important to take the ambient temperature into account. This can be done by letting the estimated time-delay and the model parameters vary with ambient temperature, for example, by approximating that relationship between the model parameters, i.e. time-delays, zeros and poles, and the ambient temperature by low-order polynomials.

After performing various simulations, it became evident that it is not always possible to find a good relationship between the model parameters and the ambient temperature. The variations of the model parameters for different ambient temperatures were too large. For instance, ambient temperature variations between winter and summer periods can be quite large. Therefore, also the heat loss of the column can differ a great deal, and, as a consequence, the performance of the column.

We observe that the operation of the column becomes more or less constant when compensating for the heat losses due to ambient temperature, see Fig. 3. The heat losses are compensated by adjusting the reboiler power such that the difference between the power in the reboiler and the heat loss over the column is constant with respect to the variations in the ambient temperature, see Fig. 4. The difference between the reboiler power and the heat losses is also called the effective separation heat. It is defined by $\Delta Q$, i.e. $Q_{\text {reboiler }}-Q_{\text {loss }}=\Delta Q$, throughout this paper. Thus, we use a reparametrization of the model, from $\mathcal{M}\left(Q_{\text {loss }}\left(T_{\text {ambient }}\right), Q_{\text {reboiler }}\right)$ to $\mathcal{M}\left(Q_{\text {reboiler }}-\right.$ $\left.Q_{\text {loss }}\left(T_{\text {ambient }}\right)\right)=\mathcal{M}(\Delta Q)$ with $\Delta Q$ naturally depending on the ambient temperature $T_{\text {ambient. In Section III, the depen- }}$ dence of the system on the $\Delta Q$ will be shown by compensating the variations of $Q_{\text {loss }}\left(T_{\text {ambient }}\right)$ in the reboiler in the first order, such that $\Delta Q$ becomes constant. Hence, by incorporating $\Delta Q$ in our parametric model it becomes possible to extract more accurate system models.

Thus, the proposed solution in this paper is to let the parameters, time-delays $\tau_{i}$, zeros $z_{i}$ and poles $p_{i}$, of the distillation

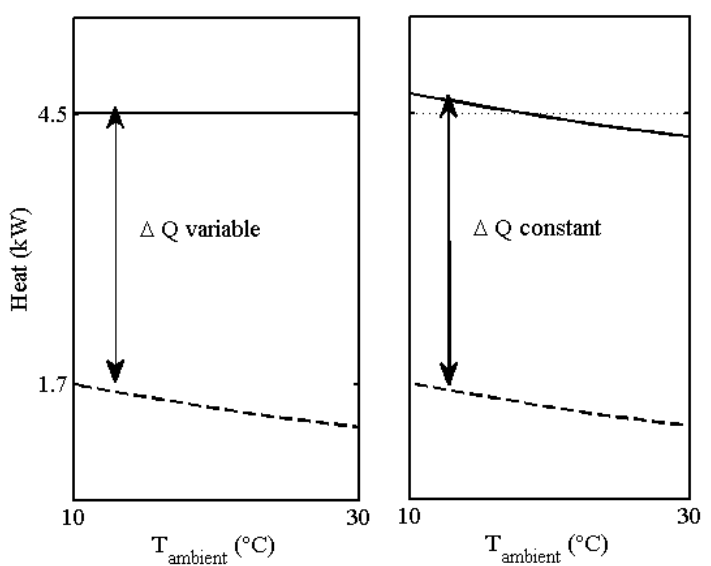

Fig. 4. Plot of the reboiler power (solid line) vs. heat loss over the column (dashed line); $\Delta Q$ denotes the difference between the power in the reboiler and the heat loss over the column. Left: $\Delta Q$ is changing with ambient temperature; right: heat losses are compensated in the reboiler power to make $\Delta Q$ constant with respect to the variations in the ambient temperature.

column model depend on the heat inside the column $\Delta Q$ :

$$
\hat{G}(s, \Delta Q)=\exp (-\tau(\Delta Q) s) \frac{\prod_{i=1}^{n_{z}}\left(s-z_{i}(\Delta Q)\right)}{\prod_{i=1}^{n_{p}}\left(s-p_{i}(\Delta Q)\right)},
$$

where $\hat{G}(s, \Delta Q)$ denotes an estimate of the FRM in continuous Laplace domain $s$ and where $n_{z}$ and $n_{p}$ denote the number of zeros and poles, respectively, with $n_{p}>n_{z}$.

\section{Simulation RESUltS}

This section shows the modeling results on a white-box model of a binary distillation column ([8], [9]) using frequency domain identification methods. The column, see Fig. 5, consists of 38 stages: a reboiler, 36 trays and a condenser. In this project, we consider a MIMO distillation system with: three inputs (the reboiler power, feed flow into the column, and reflux flow) and two outputs (temperatures in the reboiler and at the top of the column).

First, the nonparametric modeling results are compared using fast and robust LPM's in Subsection III-A. For simplicity, the ambient temperature is kept constant at $20^{\circ} \mathrm{C}$ during this simulation to clearly see the differences between the two LPM's. Also, a distinction is made between a SIMO case and a MIMO case. In Subsection III-B, the nonparametric FRM and its total variance bounds are used to find a parametric rational-form Laplace model. The results are shown for various constant levels of ambient temperature. Also, the results of compensation for the variations in $Q_{\text {loss }}\left(T_{\text {ambient }}\right)$ are shown.

\section{A. Fast vs robust LPM}

In this subsection, the nonparametric FRM's and their total variances are compared using both the fast and the robust LPM's. Although the noise and nonlinear distortions can still 


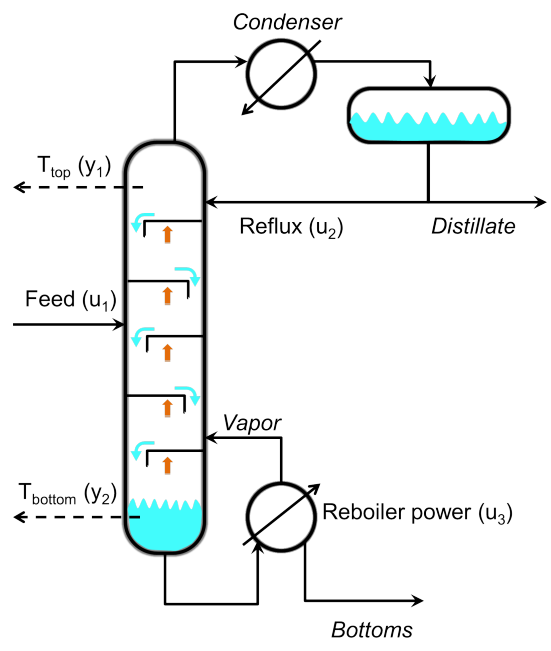

Fig. 5. A schematic representation of a binary distillation column. Here we consider three inputs: feed flow, $u_{1}$, reflux flow, $u_{2}$, and reboiler power, $u_{3}$, and two outputs: temperature at the top, $y_{1}$, and temperature at the bottom of the column, $y_{2}$.

be separated in this framework, we consider here the total variance (see Section II). The ambient temperature is kept constant during one simulation, such that we have a timeinvariant system. In Subsection III-A1 we consider three SIMO systems, from each of the three inputs to the two output temperatures. In Subsection III-A2 we consider a MIMO system with three inputs and two outputs.

1) Single-Input-Multiple-Output case: We consider one by one the three SIMO systems from the inputs feed, reboiler power and reflux to the two temperature outputs. Here we focus only on the influences of the three inputs on the temperature at the bottom. We use one realization of a randomphase multisine excitation for the fast LPM and two required realizations for the robust method, both eight periods long. One period is one hour for the robust method with a sample period of 1 second. To have the same signal-to-noise ratio's for both methods the total measurement time has to be equal. Thus, one period of the excitation for the fast method is twice as long, namely two hours, as one period for the robust method. The following harmonics are excited: lines 1 to 60 and the odd lines from line 60 to 120, i.e. [1:1:60 61:2:120]. The multisine is chosen to be full in the low frequency range, lines 1 to 60 , to have a large number of low frequency spectral lines; only the odd lines in the higher frequency, odd lines from 61 to 120 , are excited to be able to detect nonlinear distortions.

Results are shown in Fig. 6, left column. The nonparametric FRM's and their variances using both LPM's are presented. For all the three input-output pairs the robust FRM coincide well with the fast FRM, except in the lower-frequencies. This becomes clear by looking at the variance of the estimates. For equal polynomial orders, the fast method gives an FRM with a higher variance in the low-frequency region that the robust method.

Fig. 6(a) presents the nonparametric FRM and its variance from the feed to the temperature at the bottom. Because the frequency response in the low-frequency range is steep, a polynomial of order 2 of the fast LPM gives a bad result due to undermodeling [15]. Taking a higher order polynomial gives better results for the low-frequency range. A polynomial of order 10 is sufficient to get a similar result as with the robust LPM. Thus, we can achieve the same quality of an FRM estimate as the robust LPM by using the fast LPM with a high order polynomial, but twice as high frequency resolution. Fig. 6(c) presents the nonparametric FRM and its variance from the reboiler power to the temperature at the bottom. The same reasoning as for the previous input-output pair holds here as well. Fig. 6(e) presents the nonparametric FRM and its variance from the reflux to the temperature at the bottom. The second order polynomial of the fast method results in an FRM with relatively high variance. Nevertheless, a polynomial of order 12 solves this problem.

2) Multiple-Input-Multiple-Output case: In this subsection we use a simulation with an orthogonal multisine excitation on all three inputs for a MIMO simulation. Again, only the results of the estimation from the inputs to the temperature at the bottom are presented. The simulation time for both fast and robust method are the same. Thus, the fast method uses a period length of six hours and eight periods. The robust method uses a period length of one hour of an orthogonal multisine as in Eq. (1) with $i=3$ and $r=2$. This means that fast method gives an RFM estimate with six times higher frequency resolution as the robust method.

Fig. 6, right column, presents the nonparametric FRM's and their variances for a MIMO simulation data. As in the SIMO case, there is undermodeling in the low-frequency region when a polynomial of low order is used for the fast LPM. A higher order polynomial, in this case order 10, reduces the undermodeling error in magnitude, but sometimes the phase of the estimated FRM starts to show an oscillating behavior, see Fig. 6(d). The reason for this is that the size of the matrix $K_{n}$ increases with the order of the local polynomial (see Subsection II-B1). For high polynomial orders, the matrix $K_{n}$ can become ill-conditioned. Fig. 7 shows the condition number with increasing order of polynomial, starting with order 2. For every increase of polynomial order by two, about one decimal of precision is lost. Even more so at the borders, where the undermodeling effect is the largest. For example, a polynomial of order ten decreases the accuracy of the estimated FRM by five decimals, and at the borders by eight decimals.

In conclusion, for SIMO type systems we can use the fast LPM, because it results in two times higher frequency resolution and gives comparable results to the robust LPM. By taking a higher order polynomial it is possible to reduce the undermodeling effect in the frequency regions where there are large variations in the frequency response.

For MIMO systems it becomes more difficult to fit a polynomial to a FRM where the variations in frequency response are large. More qualitative research needs to be done to find out the limits of the fast LPM. Nevertheless, the fast method gives a higher resolution than the robust method when using 

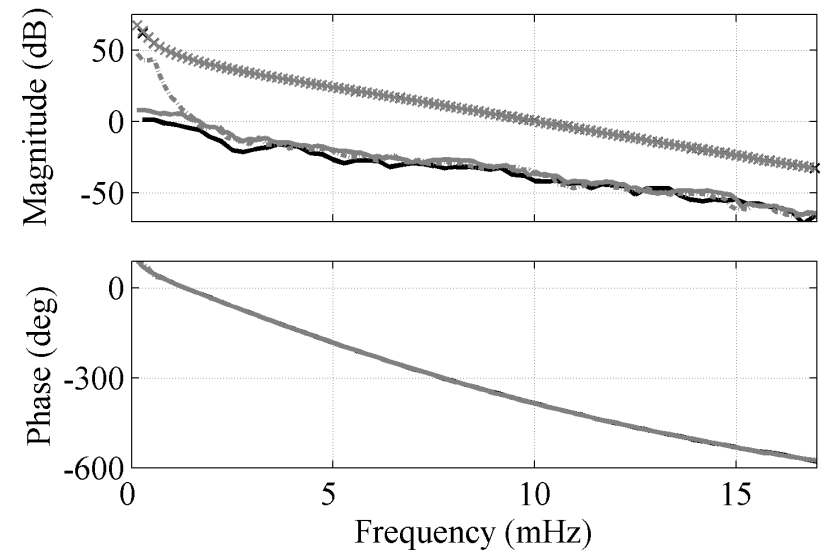

(a) SIMO estimation: excited input is feed. (solid gray) Fast LPM with polynomial of order 10 .
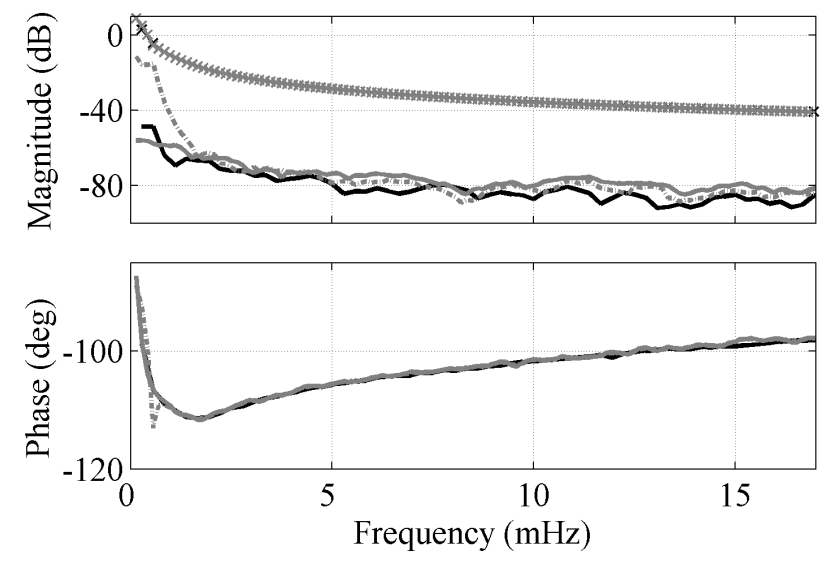

(c) SIMO estimation: excited input is reboiler power. (solid gray) Fast LPM with polynomial of order 10 .
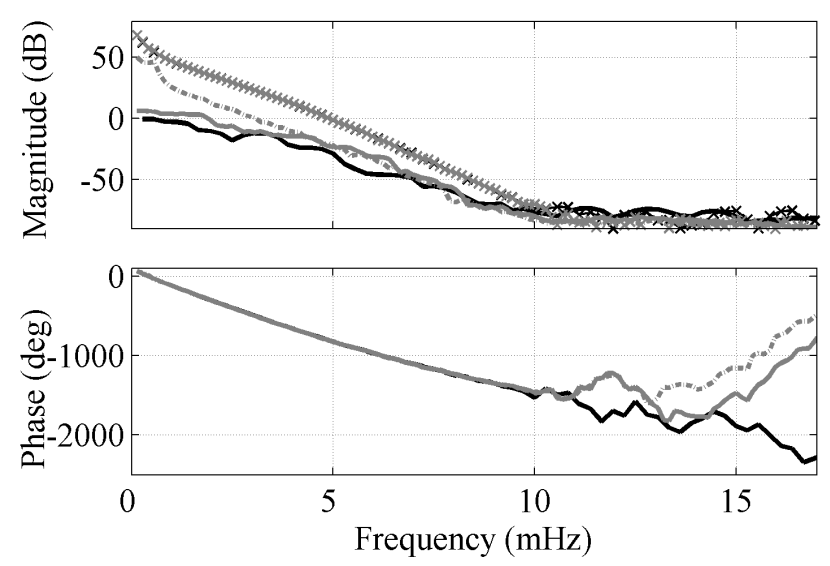

(e) SIMO estimation: excited input is reflux. (solid gray) Fast LPM with polynomial of order 12 .
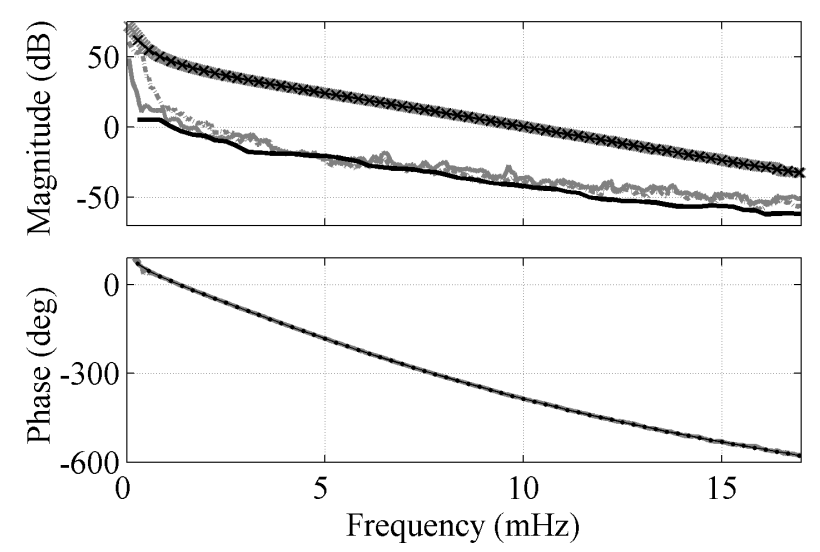

(b) MIMO estimation: from feed to temperature at the bottom.
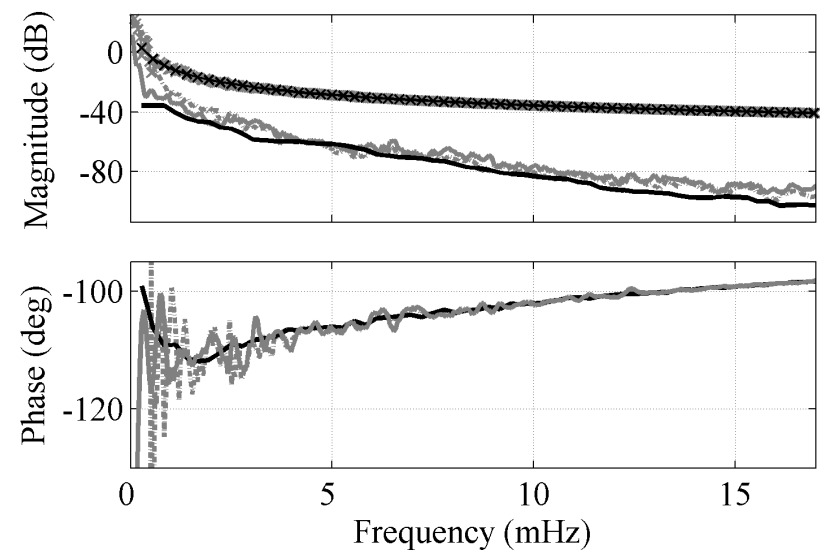

(d) MIMO estimation: from reboiler power to temperature at the bottom.
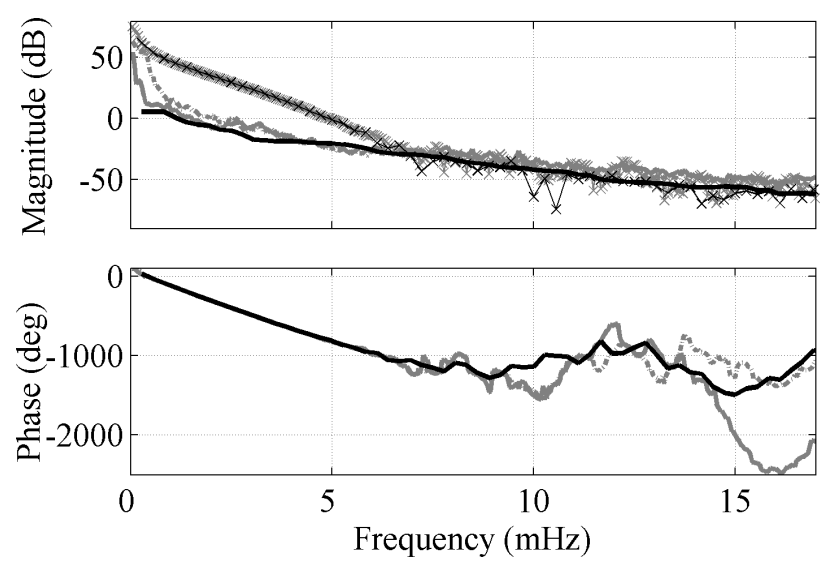

(f) MIMO estimation: from reflux to temperature at the bottom.

Fig. 6. a), c), e): The nonparametric FRM and its variance from all the three inputs to temperature at the bottom, using SIMO simulation data. (x) Nonparametric FRM's using fast (gray) and robust (black) method; (solid black) variance of the robust LPM FRM estimate; (dashed-dot gray) variance of the fast LPM FRM estimate with polynomial order 2 and (solid gray) with polynomial order 10 or 12. Thus, taking the degree of polynomial for the fast LPM large enough, the variance of the FRM becomes similar to the variance of the robust LPM FRM estimate.

b), d), f): The nonparametric FRM and its variance from all the three inputs to temperature at the bottom, using MIMO simulation data. (x) Nonparametric FRM's using fast (gray) and robust (black) method, frequency resolution of fast LPM is here six times larger than of robust LPM; (solid black) variance of the robust LPM FRM estimate; (dashed-dot gray) variance of the fast LPM FRM estimate with polynomial order 2 and (solid gray) with polynomial order 10 . 


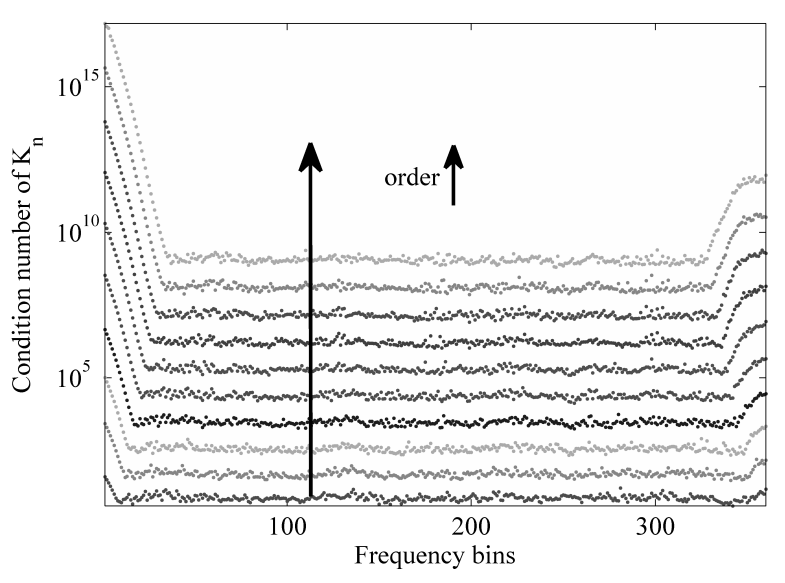

Fig. 7. Condition numbers of the matrices $K_{n}$ of the fast LPM are presented for several frequency bins. The size of $K_{n}$ data matrix is dependent on the degree of the local polynomial. In this plot, the polynomial orders are increased from order 2 by a degree of two. Condition number is shown on a log-scale and it is directly related with the accuracy of the FRM estimate. Thus, increasing the order of polynomial by two makes the accuracy of the FRM estimate decrease by one decimal.

measurements of equal size (in case of MIMO system).

In the rest of the paper we use only the robust LPM to find a nonparametric FRM with its variances in a MIMO case, because of the ease of usage.

\section{B. Constant levels of ambient temperature}

The model of the distillation column is extracted for a constant ambient temperature level from 10 to $30{ }^{\circ} \mathrm{C}$, $T_{\text {ambient }} \in[10,30]{ }^{\circ} \mathrm{C}$. For each ambient temperature, two realizations of the system's response are simulated and within each realization there are three experiments with orthogonal multisine excitations. The transient responses of these simulations are processed with the robust LPM to get a nonparametric estimate of the FRM together with its (total) variance bounds. In the final step, the nonparametric FRM and its variance bounds are used to find a parametric rational-form Laplace model of maximum order four with a time-delay term.

The frequency domain continuous-time Laplace models are compared for cases when $\Delta Q$ varies and when $\Delta Q$ is constant. Fig. 8 shows one of the poles of the third order rational model from reboiler power $u_{3}$ to the temperature at the bottom $y_{2}$. The fitted parametric model has zero delay. The 95\%confidence bounds of the poles are indicated in the figure by solid lines.

Fig. 8 shows the relations between the pole and $\Delta Q$, between $\Delta Q$ 's and the ambient temperature, and between the pole and the ambient temperature. The plot in the top right corner is related to Fig. 4, except that the actual difference between the reboiler power and the heat loss over the column, $\Delta Q$, is shown. For a constant reboiler power, $\Delta Q$ varies with the ambient temperature and consequently, the pole varies with the ambient temperature. This is shown in Fig. 8 at the bottom (marked with a cross). When the reboiler power is adjusted such that the difference between the power in the reboiler and
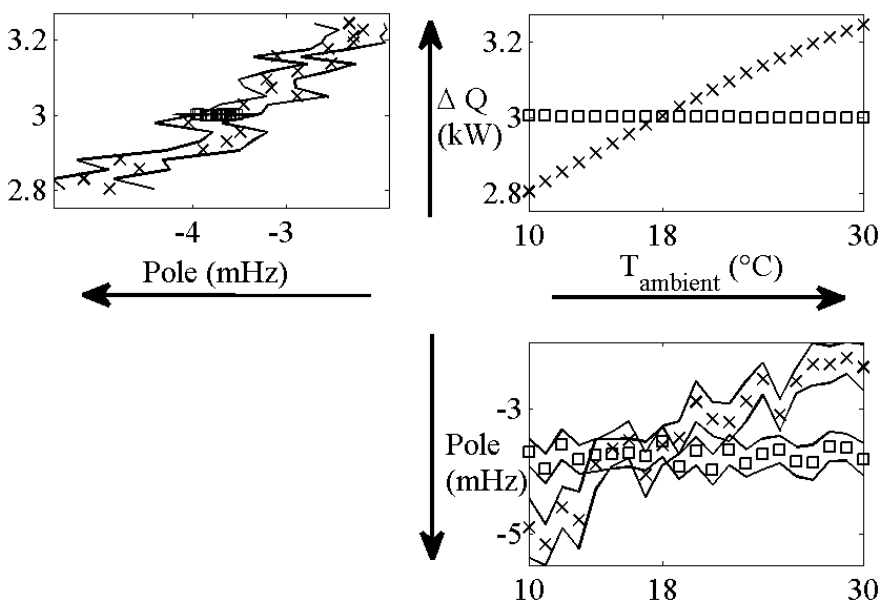

Fig. 8. Plot of one of the poles of the parametric model, together with its $95 \%$-confidence bounds, from reboiler power $u_{3}$ to the temperature at the bottom $y_{2}:(\times) \Delta Q$ varies with the ambient temperature, ( $\left.\square\right) \Delta Q$ is constant. Top left: relation between pole and $\Delta Q$; top right: estimated $\Delta Q$ 's depending on the ambient temperature (this plot is related to Fig. 4); bottom: relation between the pole and the ambient temperature.

the heat loss over the column is independent of the ambient temperature, i.e. $\Delta Q$ is contant, the pole as a function of ambient temperature becomes more or less constant, see Fig. 8 at the bottom (marked with a square). This means that the model of the distillation column becomes almost independent of the ambient temperature. We also observe this in the top left plot of Fig. 8: for $\Delta Q$ varying with ambient temperature, the pole also varies; for constant $\Delta Q$, the pole stays between $-4 \cdot 10^{-3}$ and $-3.5 \cdot 10^{-3} \mathrm{~Hz}$ for all the ambient temperatures. Consequently, it becomes easier to estimate a metamodel of the distillation column whose parameters depend on the effective separation heat $\Delta Q$.

\section{Measurement Results}

The experimental setup involves a computer-controlled packed distillation column. The column is about $6 \mathrm{~m}$ high and has an internal diameter of $6 \mathrm{~cm}$. The column works under atmospheric conditions and contains three sections of about $1.5 \mathrm{~m}$ with Sulzer CY packing (Sulzer, Winterthur) responsible for the separation. This packing has a contact surface of $700 \mathrm{~m}^{2}$ per $\mathrm{m}^{3}$ of column's volume and each meter packing is equivalent to 3 theoretical trays. The feed stream containing a mixture of methanol and isopropanol is fed into the column between packed sections 2 and 3 . The temperature of the feed can be adjusted by an electric heater of maximum $250 \mathrm{~W}$. At the bottom of the column a reboiler is present containing two electric heaters of maximum $3000 \mathrm{~W}$ each. In the reboiler, a part of the liquid is vaporised while the rest is extracted as bottom stream. At the top of the column a total condenser allows the condensation of the entire overhead vapor stream, which is then collected in a reflux drum. A part of the condensed liquid is fed back to the column as 
reflux, while the remainder leaves the column as the distillate stream. All temperatures are measured in degrees Celsius. The actuators and sensors are connected to a Compact Fieldpoint (National Instruments, Austin) with a controller interface cFP2100 and I/O modules cFP-AIO-610, cFP-AIO-610 and cFPAI-110. A Labview (National Instruments, Austin) program has been developed to control the actuators and to register the variables.

In this setup we manipulated only the reboiler power, $u_{3}$ in Fig 5. The input on the reboiler is a random multisine excitation, with a period of one hour. The other two variables, feed and reflux, were kept at a constant level. Measurements are available for the temperature at the bottom, $y_{2}$, and the temperature at the top, $y_{1}$. The ambient temperature is measured one meter from the column. The sampling period of all the data is 0.1 second.

Two experiments are done. Experiment 1: the heat losses and thus also $\Delta Q$ are varying with ambient temperature. Experiment 2: the difference in the heat losses with respect to Experiment 1 and also the variations of the heat losses with respect to the variations of ambient temperature are compensated in first order in the reboiler power. In this way, $\Delta Q$ of Experiment 2 is, on average, at the same level as $\Delta Q$ from Experiment 1. Also, $\Delta Q$ of Experiment 2 is kept constant with respect to the variations in ambient temperature.

Contrary to the simulation, we can not manipulate the ambient temperature. To overcome this problem, the measurement data of both experiments is divided and processed per two periods (by two hours). For each two periods we estimate a nonparametric FRF model together with its error bounds using the robust LPM with 1 experiment and 1 realization (see Subsection II-B). Then, using these estimates we find a loworder parametric FRF. From both measurements of 6 hours long, we got 5 intervals of data per experiment by using $50 \%$ overlap between two successive intervals.

As in Section III, we estimate an FRF from the reboiler power $u_{3}$ to the temperature at the bottom $y_{2}$. A second order rational model without delay is sufficient for both experiments. Fig. 9 shows both low- and high-frequency poles with their 95\%-confidence bounds of the fitted FRF's. The ambient temperature difference between the two experiments is, on average, more than two degrees. During the individual experiments the ambient temperature variations were less than one degree, as we can see from Fig. 9. Squares indicate the poles from Experiment 2 (where the heat losses were compensated) for all the two-periods data intervals. Crosses indicate the poles from Experiment 1.

Fig. 9 shows that the high-frequency poles (bottom) have relatively large confidence bounds for both experiments. The low-frequency poles (top) have smaller confidence bounds. This plot shows that low-frequency pole for the compensated heat loss (Experiment 2) varies less with the ambient temperature than the one for the noncompensated heat loss (Experiment 1). Comparing the poles' magnitudes between Experiment 1 and Experiment 2 shows that the poles of Experiment 2 are more or less of the same magnitude as poles

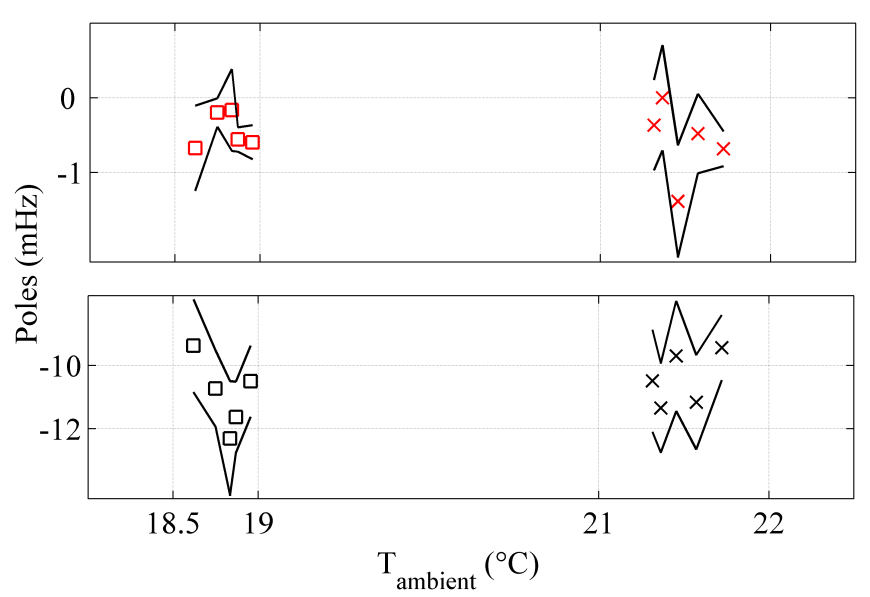

Fig. 9. Plot presents poles of a second order parametric model from reboiler power to temperature at the bottom using real measurements. The data is divided into intervals of two periods with $50 \%$ overlap. The poles of the extracted models, together with their 95\%-confidence bounds, are plotted against the mean of the ambient temperature during an interval. $(x)$ Variations of heat loss due to the ambient temperature are not compensated; ( $\square$ ) heat loss is compensated in the reboiler power for the ambient temperature variations and for the difference between heat losses of both experiments. (top) Lowfrequency poles; (bottom) high-frequency poles.

of Experiment 1. The measurement results presented here are not as conclusive as the simulation results in Section III. For comparison, see bottom plot of Fig. 8. An explanation for these differences could be, that the ambient temperature did not change much during the measurement experiments. Thus, more experiments with larger ambient temperature variations are needed to confirm the results from Section III.

\section{CONCLUSION AND DISCUSSION}

This paper applies and compares the fast and robust variations of a recently developed local polynomial method on simulations and measurements of a distillation column. These LPM's extract a good nonparametric estimate of the FRM of our system from the transient response of the system to a random-phase multisine excitation. In addition, the LPM makes it possible to estimate the variance of the nonparametric FRM in the presence of both nonlinearities and additive noise. Results show that the fast method is more efficient for a SIMO system in terms of measurement time. The robust method is better suited for MIMO systems with large variations of the frequency response on a small frequency interval, like in our case for the distillation column.

Furthermore, we have identified a parametric model with parameters depending on the difference between the reboiler power and the heat loss of the column, $\Delta Q$. We found that the operating point of the column is more or less the same when keeping $\Delta Q$ constant with respect to the variations in the ambient temperature. The results were illustrated using both simulation data and using real measurements of a pilot-scale binary distillation column at KULeuven-BioTeC. Thus, by keeping the operating point of the system constant, it becomes 
easier to estimate the relation between the system and the ambient temperature. Future work will contain an extraction of a metamodel of the distillation column whose parameters depend on the effective separation heat varying with ambient temperature.

\section{ACKNOWLEDGMENT}

This work is sponsored by the Vrije Universiteit Brussel, dept. ELEC, Pleinlaan 2, 1050 Brussels, Belgium; Fund for Scientific Research (FWO-Vlaanderen) project FWOAL561; the Belgian Federal Government (IUAP $\mathrm{VI} / 4$ ); OT/10/035; Optimization in Engineering (OPTEC) PFV/10/002; SCORES4CHEM KP/09/005; and Belgian Program on Interuniversity Poles of Attraction, initiated by the Belgian Federal Science Policy Office.

\section{REFERENCES}

[1] S. Skogestad, "Dynamics and control of distillation columns - a critical survey," Modeling, identification and control, vol. 18, no. 3, pp. 177217, 1997.

[2] J. C. MacMurray and D. M. Himmelblau, "Modeling and control of a packed distillation column using artificial neural networks," Computers and Chemical Engineering, vol. 19, no. 10, pp. 1077-1088, 1995.

[3] H. H. J. Bloemen, C. T. Chou, T. J. J. V. D. Boom, V. Verdult, M. Verhaegen, and T. C. Backx, "Wiener model identification and predictive control for dual composition control of a distillation column," Journal of Process Control, vol. 11, no. 6, pp. 601-620, 2001.

[4] U. Mathur, R. D. Rounding, D. R. Webb, and R. J. Conroy, "Use model-predictive control to improve distillation operations," Chemical Engineering Progress, vol. 104, pp. 35-41, 2008.

[5] D. E. Rivera, H. Lee, H. D. Mittelman, and M. W. Braun, "High-purity distillation: using plant-friendly multisine signals to identify a strongly interactive process," IEEE Control Systems, vol. 27, no. 5, pp. 72-89, 2007.

[6] E. W. Jacobsen and S. Skogestad, "Identification of ill-conditioned plants - a benchmark problem," in The Modeling of Uncertainty in Control. Springer, 1994, pp. 367-376.

[7] R. Pintelon and J. Schoukens, System identification. A frequency domain approach. IEEE, 2001.

[8] M. Diehl, "Real-time optimization for large scale nonlinear processes," Ph.D. dissertation, University of Heidelberg, 2002.

[9] J. Bonilla, "Structure and convexity exploitation in nonlinear chemical process modeling and estimation," Ph.D. dissertation, Katholieke Universiteit Leuven, 2011.

[10] D. Ugryumova, G. Vandersteen, B. Huyck, F. Logist, J. V. Impe, and B. D. Moor, "Identification and modeling of distillation columns from transient response data," in Proceedings of IEEE International Instrumentation and Measurement Technology Conference, 2012, pp. 2098-2103.

[11] R. Pintelon, J. Schoukens, G. Vandersteen, and K. Barbé, "Estimation of nonparametric noise and FRF models for multivariable systems - part I: theory," Mechanical Systems and Signal Processing, vol. 24, no. 3, pp. 573-595, 2010.

[12] T. P. Dobrowiecki, J. Schoukens, and P. Guillaume, "Optimized excitation signals for MIMO frequency response function measurements," IEEE Transactions on Instrumentation and Measurement, vol. 55, no. 6, pp. 2072-2079, 2006.

[13] R. Pintelon, G. Vandersteen, J. Schoukens, and Y. Rolain, "Improved (non-)parametric identification of dynamic systems excited by periodic signals - the multivariate case," Mechanical Systems and Signal Processing, vol. 25, no. 8, pp. 2892-2922, 2011.

[14] "Frequency domain system identification toolbox for MATLAB - general information." [Online]. Available: http://matlab.gamax.hu/english/products/fdident/

[15] G. Monteyne, D. Ugryumova, and G. Vandersteen, "Extension of local polynomial method for periodic excitations," in Proceedings of SYSID 2012, to appear, 2012. 
Research Coordination Office Huis Bethlehem Schapenstraat 34 B-3000 Leuven Tel. : +3216 Belgium

Fax: +32 16324065 $\frac{\text { research@kuleuven.be }}{\text { www.kuleuven.be }}$ 